\title{
The Concept of a Regional Information-Analytical System for Emergency Situations
}

\author{
Igor Grebennik ${ }^{1}$, Oleksandr Khriapkin ${ }^{1}$, Ata Ovezgeldyyev ${ }^{2}$, \\ Valentina Pisklakova ${ }^{1}$, Inna Urniaieva ${ }^{1}$ \\ ${ }^{1}$ Kharkiv National University of Radio Electronics, Kharkiv, Nauka Ave, 14, Ukraine \\ ${ }^{2}$ Kyiv National University of Culture and Arts, Kyiv, Yevgena Konovaltsa str. 36, Ukraine \\ igor.grebennik@nure.ua
}

\begin{abstract}
The concept of a regional information-analytical system for emergency situations is presented. The three-level architecture of such a system and the functions of its main components are described. A generalized model for assessing plans for eliminating the consequences of emergencies is described. The goals and peculiarities of the structural modules for the regional information and analytical system for the prevention and elimination of emergencies are detailed; some examples of the implementation of local subsystems for Ukraine are considered.
\end{abstract}

Keywords: Disaster Response, Disaster Communication, Information Processing for Emergency Management.

\section{Introduction}

In the situations associated with modern risks of both anthropogenic and natural type leading to emergency situations, an urgent issue is the development of infor-mation and analytical system for eliminating and preventing such situations [1].

A number of requirements have to be imposed on efficiency of such systems: the minimization of both human and economic losses during the elimination of emer-gency situations; the prevention of possible emergencies in the monitoring mode as soon as possible [2-4].

All possible causes for emergency can be divided to natural and anthropogenic ones. Natural factors that can lead to emergencies can be classified as follows [5]:

- droughts;

- floods;

- earthquakes;

- tsunami;

- etc.

In this case, the natural causes have such basic characteristics as:

- complexity of warning; 
- complexity of forecasting;

- need for the development of complex prediction models.

At the same time, the following points can be considered as risks in the appearance of natural emergencies [5]:

- a large scale of destructions that often happen;

- large human, financial and infrastructural losses.

The following anthropogenic factors can be considered:

- chemical or biological pollution in enterprises;

- traffic accidents;

- military actions;

- etc.

The main characteristics of anthropogenic causes for emergencies are as follows:

- it is necessary to keep the technical passports of all technogenic objects;

- in the presence of the initial information, it is possible to construct quite accurate models;

- the probability of a warning is high.

The main risks of anthropogenic factors of emergencies are these:

- long-term consequences (pollution of the territory) are possible;

- large human, financial, infrastructural losses;

- a large multiplier effect on the economy is possible.

\section{Problem formulation}

The consideration and solution of such problems and tasks separately are ineffective as it doesn't allow taking into account all the interrelations between factors of occurrence of emergencies themselves and their consequences. Besides, the separate solution to each such a task can lead to an inefficient use of the available forces and it means eliminating the consequences of emergencies as well as a poor coordination of services and departments. Thus, the creation of a concept of an information and analytical system for preventing and eliminating the consequences of emergency within the region and the country in general is relevant $[4,6,7]$.

For each of the possible causes of an emergency situation, the information and analytical system should provide the opportunity to address the following issues:

- prevention of an emergency;

- timely response to an emergency situation, if it occurs;

- the formation of an effective emergency response plan through the coordination of all departments of forces and assets;

- the coordination of the plan of eliminating the consequences of an emergency;

- the formation of conclusions on an emergency situation and taking them into account in the future. 
The main functions of such a system are the following:

- the registration of messages and emergency situations;

- the classification of emergencies;

- the certification (monitoring) of potentially dangerous objects;

- conducting directories;

- geoinformation support;

- creating reports;

- modeling (the forecasting of emergencies).

\section{Solution}

Emergency prevention and elimination in Ukraine is engaged in State Emergency Service of Ukraine (SESU). The structure of the SESU consists of such units [8]:

- rescue units of special purpose;

- units of the technical service;

- state fire-fighting units;

- supply centers;

- operational coordination centers;

- centers for operational communications, telecommunications systems and information technologies.

\subsection{Goals and objectives of the Regional Information-Analytical System for Emergency Situations}

The Regional Information-Analytical System for Emergency Situations (RIASES) is an integral part of the program for informatization and a unified state system for the prevention and elimination of accidents, catastrophes and other emergencies.

The purpose of creating RIASES is to provide the operational executive authorities with expert analytical, predictive, reference, statistical, factual, control, reporting and management information by using modern information technologies for solving problems related to technological and environmental safety and emergency situations.

The main tasks of creating RIASES are the following:

- providing the central executive authorities, the regional and district state administrations with reliable information related to man-caused and environmental safety and emergency situations;

- ensuring compatibility of information on emergency situations through the use of unified principles for building databases, unified certified cartographic information, common classifications and standards;

- obtaining a research-based forecast of the possible occurrence of an emergency and its possible consequences;

- providing operational access to information resources; 
- obtaining reasonable recommendations as to a rational allocation and using of the available resources for the elimination of emergency consequences;

- ensuring information exchange and coordinating the actions of the executive authorities in preventing the occurrence of emergencies or the elimination of its consequences.

The goal is achieved by solving the following main tasks:

- system-wide:

- the analysis of the technology for information exchange between the executive authorities responsible for treating emergencies;

- the development of an architecture for RIASES, the description of its structural elements;

- determining information flows in RIASES;

- the development of mechanisms for information exchange within RIASES;

- defining requirements for RIASES;

- the development of specifications for software and hardware of RIASES and the installation of the technical facilities and the system software, the establishment of telecommunications between the structural elements of RIASES;

- the creation of a technological stand for RIASES, the development of its main functions and their phased implementation;

- functional:

- determining the main functions that should be implemented in the RIASES;

- determining, setting and developing of a set of functional tasks, the solution of which will be carried out at different levels of RIASES;

- The development of regulatory and legal support.

\subsection{The main functions and tasks of RIASES}

In order to achieve the above goals, the RIASES performs the main functions, which are grouped into four groups:

- informing;

- analysis and forecasting;

- planning activities and preparing solutions;

- monitoring the implementation of decisions and activities.

The implementation of the basic functions of these generalized groups provides the solution to the following series of problems.

The tasks of the "Informing" group.

To implement the functions of the "Informing" group it is supposed to resolve the following main problems using the RIASES elements: 
- the automation of the processes for obtaining complete and reliable information on emergencies in the territory of Ukraine at all levels of RIASES;

- informing the management of the central and local executive authorities and other organizations, if necessary, about emergencies that have occurred, the threat of appearing new emergencies, the elimination of the consequences of the emergency, the damage caused, etc. according to the regulations;

- a rapid automated access of relevant experts and analysts of the subdivisions of ministries, departments, and regional state administrations to priority information on emergencies.

The tasks of the group of functions "Analysis and Forecasting".

Regarding the group of functions "Analysis and forecasting," their implementation involves solving the following generalized problems:

- aggregation data of emergency and the prerequisites for their occurrence, which will get into the RIASES and accumulate in the databases;

- define an expert evaluation of the nature of the corresponding emergency situations and the necessary resources to eliminate their consequences;

- background analysis and prediction of emergencies;

- analysis and modeling of the consequences of certain types of emergencies that occurred and the modeling of their impact on the possibility of other (derivatives) emergencies;

- predicting the impact of the most dangerous potentially possible emergencies;

- formation of regular reports on specific emergencies for the management.

The information analysis should provide for the initial stage of transformation of documentary and other important information and extracting the most essential information. Information synthesis should be connected with the process of generalization of the information obtained as a result of the information analysis of documents and the preparation of aggregation results in the text (or other) form. Depending on the nature and objectives of the work performed, the results of the synthesis can be different: from the simplest (specific description, abstract) to more complex ones (inspection, classification, selection of facts).

Task of group functions "Planning activities and preparing solutions".

Implementation of the RIASES functions of the group "Planning actions and preparing solutions" requires the solution of the following main tasks:

- the preparation of draft decisions on the planning of measures to eliminate the consequences of emergencies;

- the formation of expert information on the basis of analyzing the information from the database of precedents for specific emergencies;

- determining the criterion for the assessment of necessary resources and the development of plans that will be followed during the elimination of the consequences of emergency situations. 
The tasks of the group of functions "Monitoring the implementation of decisions and activities".

In turn, to implement the functions of the group "Monitoring the implementation of decisions and activities," the following tasks must be accomplished:

- monitoring the elimination of the consequences of emergencies;

- monitoring the implementation of the decisions that should be made as a result of resolving problems related to emergencies;

- monitoring the implementation of scheduled-preventive measures to prevent emergencies.

\subsection{Model}

The problem of predicting possible emergencies and planning their elimination is the most difficult but at the same time the most important one. This is due to the fact that in the case of a correct plan of actions for eliminating an emergency, there is a high probability of the timely response to the emergency situation and the minimization of possible losses. In case of the correct identification of the scenario for the emergency, it is possible to prevent all possible consequences.

In addition, by practicing repeatedly developed plans of eliminating possible emergencies during exercises, it is possible to improve efficiency of staff and improve the coordination of actions for services and departments.

The complexity of this task is conditioned by incomplete information in the event of natural disasters. The most effective way to solve such problems is to simulate the appearance of emergencies and the formation of possible plans for their elimination. Modern information technologies make it possible to create such simulation plans and evaluate their effectiveness during acceptable time.

At the same time, there is a relevant problem of evaluating the entire set of created plans for eliminating emergencies and selecting the most effective ones. Such a task is not trivial as it is necessary to take into account a number of conflicting characteristics (criteria). Therefore, the use of multifactor estimation and selection of optimal plans is proposed [9-11].

Generally, the problem of multicriteria optimization can be presented as follows:

$$
x^{0}=\underset{x \in X}{\arg } \operatorname{extr} F(K(x), \Lambda)
$$

where $\mathrm{X}$ is a set of permissible plans (including aspects of the emergence, prevention or elimination of emergencies);

$K(x)=\left\{k_{1}(x), \ldots, k_{n}(x)\right\}$ is a set of local criteria for evaluating plans;

$\Lambda=\left\{\lambda_{1}, \ldots, \lambda_{n}\right\}$ are numerical characteristics of the importance of local criteria.

An additional condition for the characteristics of importance is the following:

$$
\sum_{i=1}^{n} \lambda_{i}=1
$$


Local criteria in many aspects depend on the specific type of emergency, but at the same time it is possible to allocate the general ones that characterize any kind of emergency:

- minimum of costs for the elimination of possible emergency consequences:

$$
C=\sum_{j=1}^{m} c_{j} \rightarrow \min ,
$$

where ${ }^{c} j$ is the cost of $\mathrm{j}$-th activity for eliminating the consequences of emergencies.

- minimum possible economic losses after the implementation of this plan

$$
U \rightarrow \min ,
$$

- minimum time spent on eliminating potential emergency consequences

$$
T \rightarrow \min ,
$$

where $T$ is a complex parameter that can take into account both sequential and parallel actions for emergency elimination.

- minimum of possible human losses in the form of wounded persons and the victims

$$
L=\sum_{q=1}^{v} l_{q}+\sum_{h=1}^{s} l_{h} \rightarrow \min ,
$$

where $v$ is the number of possible wounded persons and $s$ is the number of possible victims.

Since the criteria are heterogeneous and have different dimensions they can be transformed to a uniform dimension by means of one of the normalization types, for example

$$
\left(\frac{k_{i}(x)-k_{i}^{-}}{k_{i}^{+}-k_{i}^{-}}\right)^{\alpha_{i}}
$$

where $k_{i}(x)$ is the value of the $\mathrm{i}$-th local criterion;

$k_{i}^{-}$is the worst value of the $\mathrm{i}$-th criterion;

$k_{i}^{+}$is the best value of the i-th criterion;

$\alpha_{i}$ is a parameter determining the variant of dependence ( ${ }^{\alpha_{i}}=1$ is linear, $0<\alpha_{i}<1$ is concave, ${ }^{\alpha}>1$ is convex). 


\subsection{Organizational and functional structure of RIASES}

Architecturally, RIASES represents three-level structure (fig. 1). Each level resolves its own problems, based on the scale of the arising emergencies and existing forces, and the means at this level to eliminate it.

According to the organizational and functional characteristics, RIASES will include the following structural elements (fig. 2):

- central subsystem of RIASES;

- functional subsystems of the central executive authorities;

- territorial subsystems of the local executive authorities;

- remote functional units or remote workstations of enterprises and organizations;

- providing the subsystems of RIASES.

The general organizational and functional structure of RIASES is shown in fig. 2. The first four structural elements of RIASES perform the basic functions of RIASES, therefore they are the functional and structural elements of RIASES.

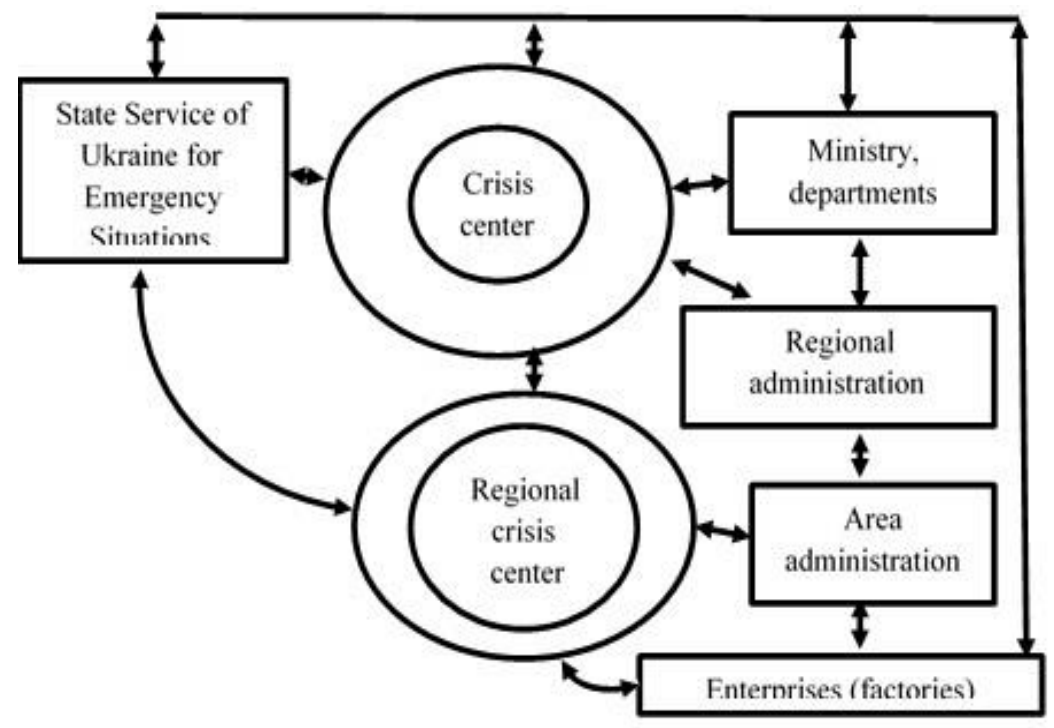

Fig. 1. Three-level structure of RIASES

Subsystem of monitoring potentially dangerous territories and objects.

- maintaining the directory of hazardous substances;

- monitoring the main sources and types of danger for objects;

- monitoring dangerous objects, structures and territories of the region;

- monitoring chemical hazards in the region;

- monitoring the hydrodynamic danger of the region;

- monitoring forces, assets and technical base. 


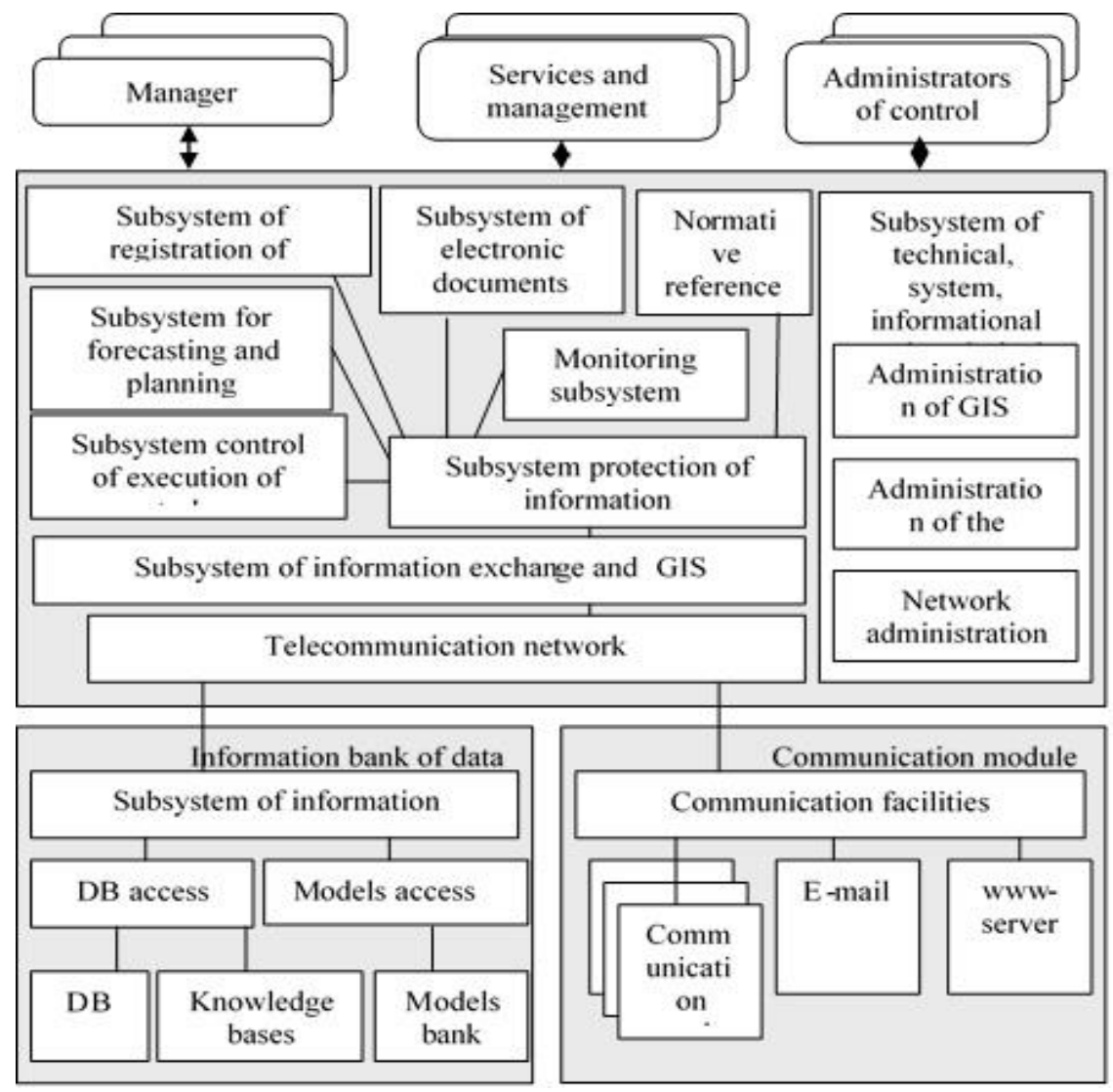

Fig. 2. Structural-functional scheme of RIASES

System for the preparation and control of decisions made

- assessment of the forces and facilities necessary for the localization of emergencies.

- forming possible sources for attracting the necessary forces and resources.

- assignment of forces and means by criteria (a promotion time minimum, minimum costs, by a generalized criterion).

- assessment of possible consequences of emergencies.

- determination of the level of the involved forces and assets, and their costs.

Function of certification (monitoring) of objects of potential danger

The basic information that should be stored in the certificate of the technical object is shown in Fig. 3. 


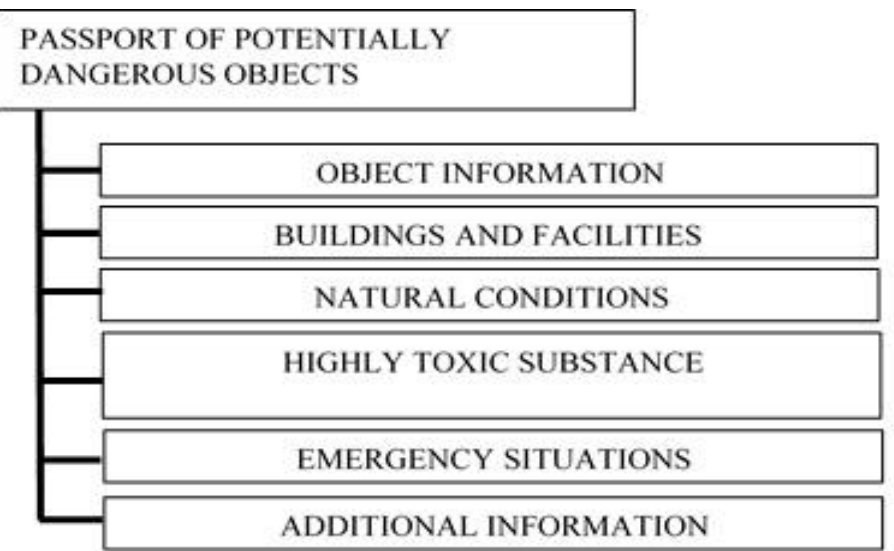

Fig. 3. Structure of the information of the passport for a technogenic object

\section{Geoinformation system}

The subsystem of geoinformation support is one of the most important elements of the system. The main task of this subsystem is the visualization of an operational environment. The examples of the work of such an implemented module are shown in Fig. 4 and Fig. 5

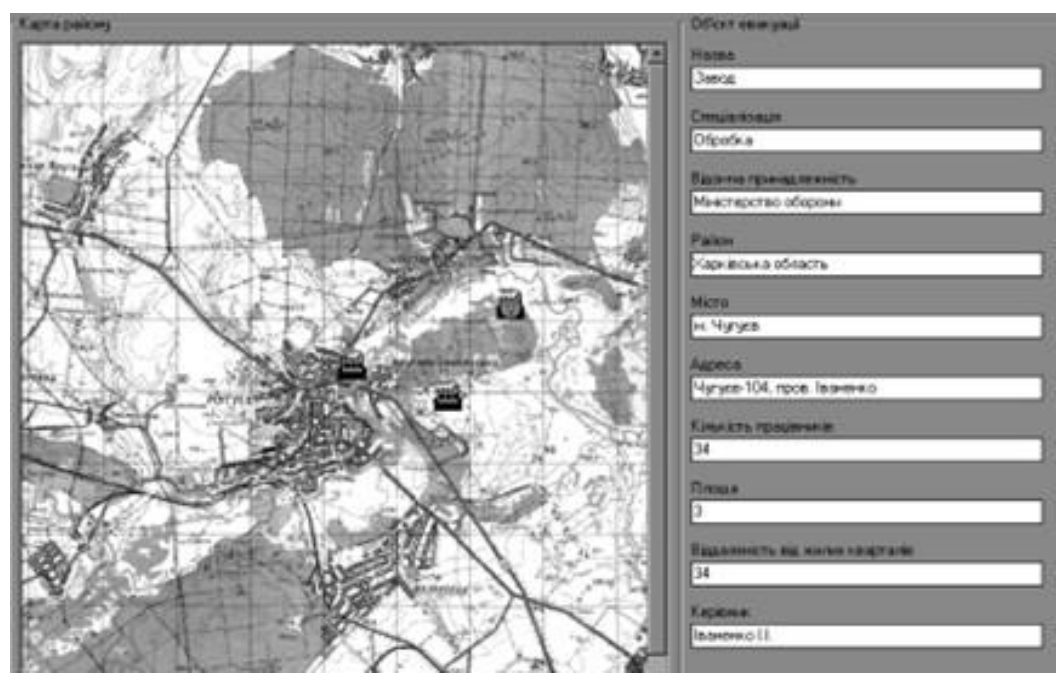

Fig. 4. - Example of an area map with identified potentially hazardous enterprises 


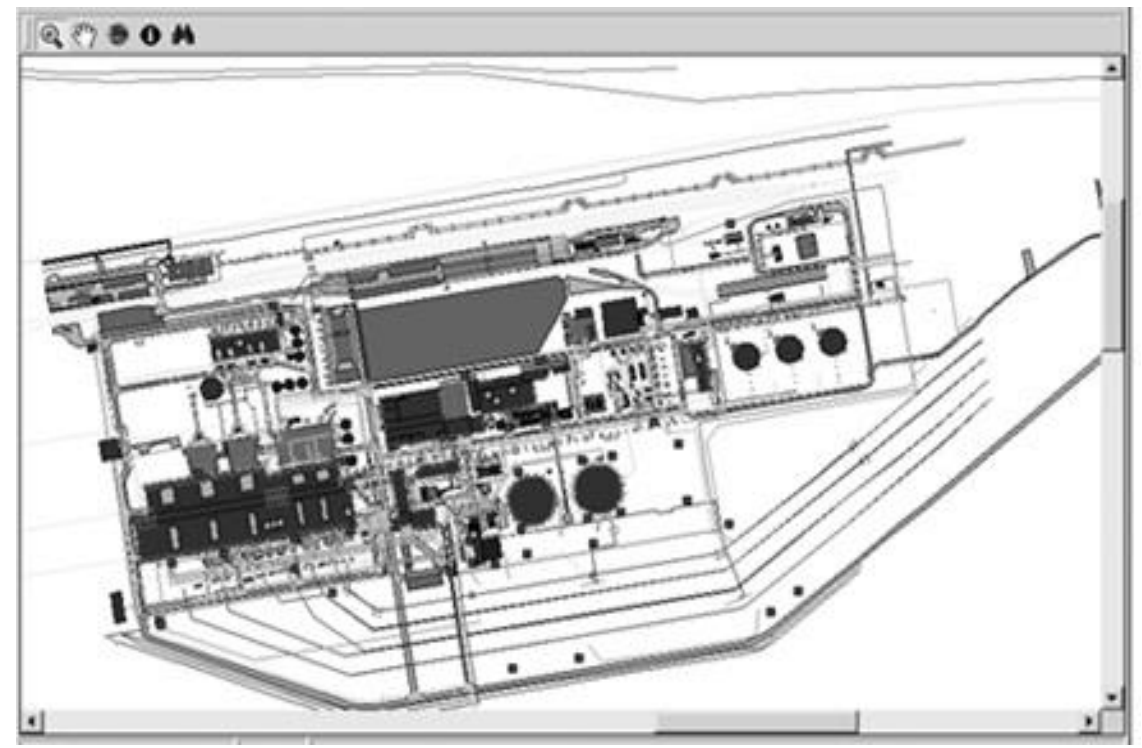

Fig. 5. - Example of the scheme of a potentially hazardous enterprise

\section{Results of the simulation of an emergency situation}

Based on the results of modeling emergencies, it is possible to analyze both numerical parameters and visualize the results (Fig.6).

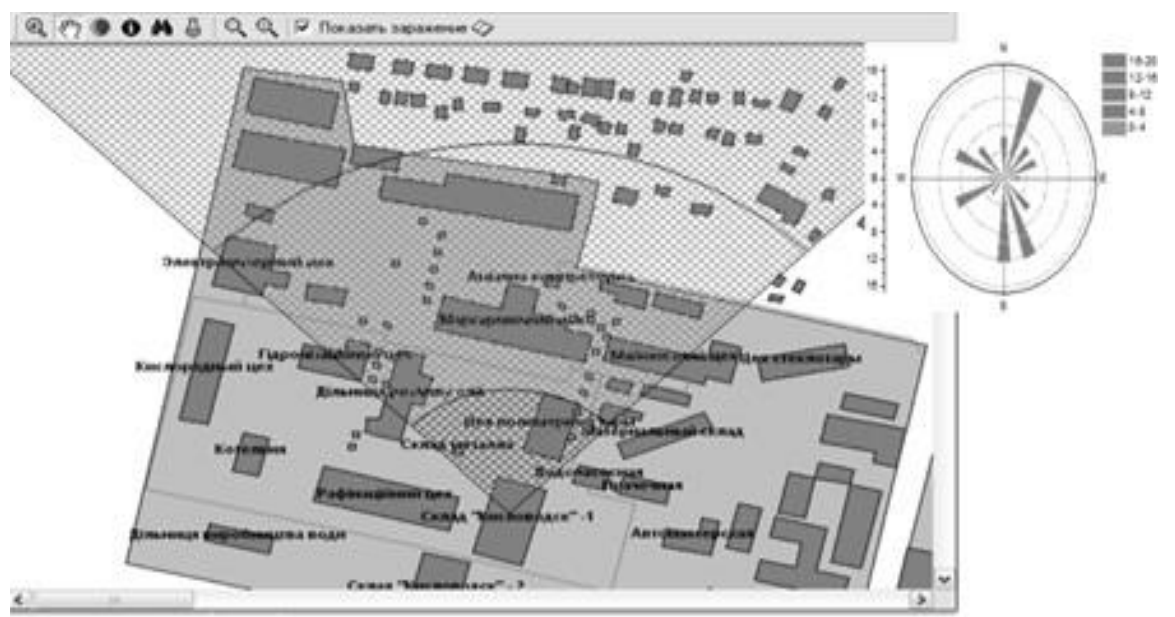

Fig. 6. - Example of simulation results for the distribution of harmful emissions taking into account wind 


\section{Conclusions}

The concept of a regional information and analytical system for the prevention and elimination of emergencies is presented. The architecture of the system and main features of its components are described, their goals and tasks being formulated. Some examples of the implementation of individual subsystems for Ukraine are considered.

\section{References}

1. Murayama, Y.,Velev, D.,Zlateva, P.,Gonzalez, J.J.: Information Technology in Disaster Risk Reduction. First IFIP TC 5 DCITDRR International Conference, ITDRR 2016, Sofia, Bulgaria, November 16-18 (2016)

2. Bartel Van De Walle, Murray Turoff, Starr Roxanne Hiltz.: Information Systems for Emergency Management. Business \& Economics. (2014).

3. C. Vecchiola, H. Anjomshoa, Y. Bernstein, I. Dumitrescu, R. Garnavi, J. von Känel, G. Wightwick.: Engineering resilient information systems for emergency management. IBM Journal of Research and Development (Volume: 57, Issue: 5, Sept.-Oct. 2013), 1-12.

4. Y. Murayama, J. Sasaki and D. Nishioka.: Experiences in Emergency Response at the Great East Japan Earthquake and Tsunami. 49th Hawaii International Conference on System Sciences (HICSS), Koloa, HI, 146-151 (2016).

5. Methods of identification of potentially dangerous objects Homepage http://zakon5.rada.gov.ua/laws/show/z0286-06/page, last accessed 2017/08/21.

6. Yumei Chen.: Research on critical success factors for Emergency Management Information System. 2nd International Conference on Artificial Intelligence, Management Science and Electronic Commerce (AIMSEC) 7186 -189 (2011).

7. Lei Song, Zong Xiao Yang, Guan Qiang Dong, Shu Guang Yu, Wen Li Yang.: Construction of expressway emergency rescue information management system using Browser/Server framework. International Conference on Networking, Sensing and Control $341-346$ (2011).

8. Structure of the State Service of Ukraine for Emergencies Homepage http://www.dsns.gov.ua/ua/Struktura.html, last accessed 2017/08/21.

9. Greco, Salvatore, Ehrgott, Matthias, Figueira, José Rui.: Multiple criteria decision analysis: state of the art surveys. (International Series in Operations Research and Management Science; Vol. 233). 2nd ed. New York : Springer (2016).

10. Matthias Ehrgott.: Multicriteria Optimization. Springer-Verlag Berlin Heidelberg. (2005).

11. I.V. Grebennik, T.E. Romanova, S.B. Shekhovtsov.: Interval estimation of alternatives in decision-making problems. Cybernetics and Systems Analysis, 45(2), 253-262 (2009). 